\title{
Natural history of thyroid cancer
}

\author{
Toru Takano
}

Department of Metabolic Medicine, Osaka University Graduate School of Medicine, Suita 565-0871, Japan

\begin{abstract}
Thyroid cancers have long been considered to arise in middle age and, after their repeated proliferation, resulting in further damage to the genome, they progress to more aggressive and lethal cancers. However, in 2014, some studies were reported that might lead to a marked change in our understanding of the natural history of thyroid cancer. A high prevalence of papillary carcinoma in the young suggested that the first initiation of thyroid cancer is likely to occur in the infantile period. Such a conclusion was also supported by a very slow growth rate of papillary microcarcinomas (PMCs) in an observation trial. The proliferation rate of PMCs was negatively correlated with the age, and surgery to remove PMCs did not contribute to reduce mortality from thyroid cancer. These findings strongly suggested the existence of self-limiting cancers, which are truly malignant but do not progress to lethal cancers, for the first time in human history. The early detection of self-limiting cancers results in overdiagnosis. Ultrasonographic screening of the thyroid in the young should be avoided. Lethal thyroid cancers, whose origin is still unknown, appear suddenly after middle age. In the elderly, thyroid cancers are a mixture of self-limiting and lethal cancers; thus, when thyroid cancer is detected, careful follow-up with examination of its growth rate is required.
\end{abstract}

Key words: Thyroid cancer, Self-limiting cancer, Lethal cancer, Overdiagnosis

\section{Thyroid cancer trilogy in 2014}

Thyroid cancer has long been considered to be generated by multi-step carcinogenesis, in which cancer cells are derived from thyroid follicular cells (thyrocytes), via multiple incidences of damage to their genome, especially in oncogenes or anti-oncogenes that accelerate proliferation or foster malignant phenotypes, such as the ability to invade the surrounding tissue or metastasize to distant organs $[1,2]$. Based on data on the prevalence of clinically evident thyroid cancer, the first event of carcinogenesis has been thought to occur in middle age, and some cancer cells acquire a more aggressive phenotype after repeated proliferation, resulting in accumulating genetic alternations $[3,4]$.

In 2014, three important studies on thyroid cancer, which might lead to a marked change in the basic con-

Submitted Jan. 19, 2017; Accepted Jan. 20, 2017 as EJ17-0026 Released online in J-STAGE as advance publication Feb. 2, 2017

Correspondence to: Toru Takano, Department of Metabolic Medicine, Osaka University Graduate School of Medicine, 2-2 Yamadaoka, Suita, Osaka 565-0871, Japan.

E-mail: ttakano@labo.med.osaka-u.ac.jp

(c) The Japan Endocrine Society cept of cancer, were published. An increased number of ultrasonographic examinations of the thyroid resulted in a markedly increased number of patients with thyroid cancer in Korea after 2000 [5, 6]. Most of them were papillary microcarcinomas (PMCs), and almost all patients underwent surgical resection of the tumor. However, in spite of the increased number of thyroid surgeries, mortality due to thyroid cancer remained the same. Such surgeries are now regarded as overtreatment. This outcome, in which the removal of PMCs did not result in the prevention of death from cancer, is quite puzzling when considering the concept that thyroid cancer is generated by multi-step carcinogenesis in which long-existing PMCs show progression to acquire a more aggressive phenotype and finally turn into lethal cancers. Microscopic neck lymph node metastases present in over $60 \%$ of PMC patients [7]. Thus, PMCs are not pre-cancers but true cancers. They already possess cancerous characteristics but do not become lethal simply because they have a limited proliferation ability. These tumors can be called self-limiting cancers. These studies were the first reports that proved the existence of a large number of self-limiting cancers in the thyroid. 
The next surprising data were presented in a report from the Fukushima Health Management Survey, in which ultrasonographic screening was carried out involving almost all children in Fukushima Prefecture, Japan. Childhood and adolescent thyroid cancers had been believed to be very rare before this project; however, papillary carcinoma was found in one out of 2,700 children, and the prevalence showed a steep increase after the age of 15 [8]. These data led many researchers to consider that the initiation of thyroid cancer occurs in the infantile period. Such an idea is also supported by two previous findings. The risk of radiation-induced thyroid cancer is evident in children approximately under the age of 5 but not in adults [9, 10]. The induction of thyroid cancer by expressing oncogenes in the thyroid was only possible when they were introduced in fetal mice [11-13].

Another study published in 2014 confirmed the above. Ito et al. performed an observation trial of PMCs for decades, and found that only $8 \%$ of the observed PMCs showed significant growth within ten years [14]. This finding clearly suggested that the initiation of these PMCs occurred not in adults but in young children. Further, PMCs in young patients grew faster, and no growth was observed in PMCs in patients older than 60 . Thus, the duration-dependent progression of cancer was not observed. Furthermore, not a single patient among 1,235 cases died from thyroid cancer during this observation trial. Considering the fact that about $10 \%$ of patients die from thyroid cancer in general $[15,16]$, it is unlikely that these PMCs show progression to lethal cancers. These findings explain the overdiagnosis of thyroid cancer in Korea. The surgical resection of PMCs which do not progress to lethal cancers does not contribute to the reduction of thyroid cancer-related mortality.

These three studies, the thyroid cancer trilogy in 2014 , might be regarded as a milestone in the history of cancer research, since they reported the existence of self-limiting cancer, which is truly malignant but does not kill the patients. Before the presentation of these studies, we justified the early detection of and surgery for small cancers, because we believed that such cancers might progress to acquire more aggressive characteristics. However, for self-limiting cancers, which are estimated to comprise the majority of thyroid cancers, early diagnosis and surgery result in overdiagnosis and overtreatment, like the cases in Korea.

\section{Natural history of thyroid cancer}

Thyroid cancer was long believed to arise from thyrocytes in middle age (Fig. 1A). However, such a classical model of natural history, the late-onset multi-step carcinogenesis model, does not explain the clinical evidence presented in the thyroid cancer trilogy. Fig. 2 shows the prevalence of thyroid cancer calculated with the autopsy database of the Japan Society of Pathology [17]. The prevalence shows a steep increase from the age 15 to 34, and then remains almost constant. A large number of thyroid cancers start growing before the age of 30 , but not in middle age.

Williams proposed a new model of the natural history of thyroid cancer, called the early-onset multistep carcinogenesis model (Fig. 1B) [18]. The origin of thyroid cancer is the thyrocyte, as also proposed in the late-onset multi-step carcinogenesis model. The initiation of cancer occurs during the infantile period. After the initiation, tumor cells grow very slowly. During their repeated proliferation, some tumor cells acquire additional alternations in their genome, resulting in progression to more aggressive tumor cells. Macro carcinomas in aged patients are more aggressive since they accumulate genetic alternations after repeated proliferation over decades.

Several questions arise, however, when we try to explain clinical and experimental data on thyroid cancer using this model. As described in the previous section, there is no increased risk of thyroid cancer after radioactive iodine therapy in adults [10]. It is not possible to induce thyroid cancer by introducing oncogenes in the thyroid of adult mice [13]. It is difficult to explain these findings when thyroid cancer arises from thyrocytes. Differences in the proliferation rate can partly explain this phenomenon. However, there is a marked discrepancy between the thyroid cancer risk and proliferation rate of thyrocytes [19]. Their proliferation rate remains almost the same from the age of 0 to 10 . However, the risk of radiation-induced thyroid cancer decreases after the age of 5. A large number of thyrocytes still proliferate in young adults. However, after the administration of radioactive iodine for the treatment of Graves' disease, no increase in the thyroid cancer risk is observed [10].

In the early-onset multi-step carcinogenesis model, additional genomic alternations after the initial mutation during the infantile period turn a "benign" cancer into an aggressive one, causing death from cancer. 
A

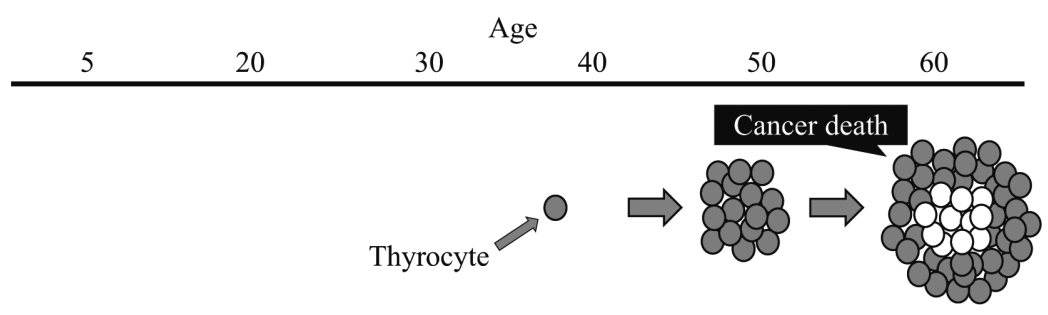

B

\begin{tabular}{|c|c|c|c|c|c|}
\hline \multicolumn{6}{|c|}{ Age } \\
\hline 5 & 20 & 30 & 40 & 50 & 60 \\
\hline Init & & Gra & & & \\
\hline
\end{tabular}

C

\begin{tabular}{llllll}
5 & 20 & 30 & Age & & \\
& & 40 & 50 & 60 \\
\hline
\end{tabular}

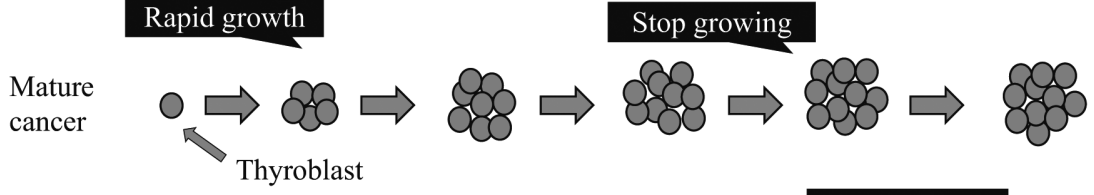

Cancer death

Immature cancer

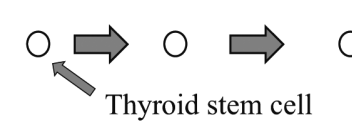

\section{Sudden growth}
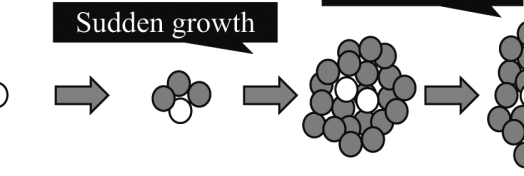

Fig. 1 Three proposed models of the natural history of thyroid cancer

A, the late-onset multi-step carcinogenesis model; $\mathbf{B}$, the early-onset multi-step carcinogenesis model; and $\mathbf{C}$, the fetal cell carcinogenesis model.

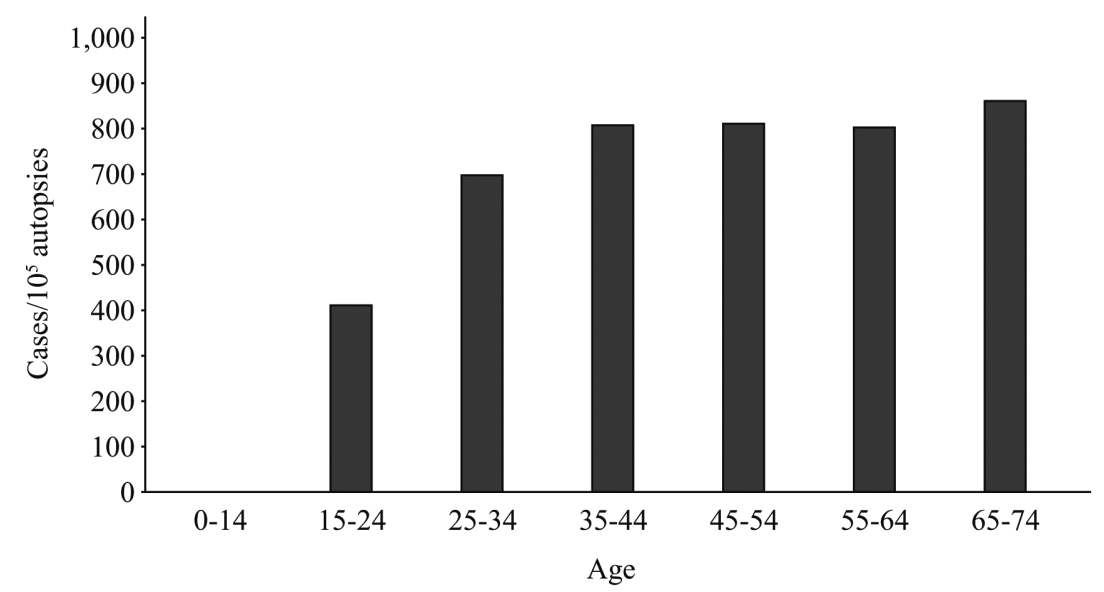

Fig. 2 Thyroid cancers detected in autopsied cases 
However, to date, the accumulation of genetic alternations in aggressive cancers, such as undifferentiated and anaplastic carcinomas, has not been reported. Rather, genetic alternations in thyroid tumors are tissue-type-specific [20-23]. Some alternations are only observed in anaplastic carcinomas, whereas others are dominant in differentiated carcinomas. Some previous studies suggested that the $B R A F$ mutation is associated with a poor prognosis in papillary carcinoma patients $[24,25]$. However, even this is controversial, since the $B R A F$ mutation was found with a high prevalence in juvenile cases on conducting the Fukushima Health Management Survey, which are expected to show an excellent prognosis [26].

One of the puzzling characteristics of thyroid cancer is the discrepancy between tumor aggressiveness and the prognosis. Thyroid cancers in young patients grow rapidly and frequently accompany distant metastasis. Even in such cases, thyroid cancers in the young are associated with an excellent prognosis and, after appropriate therapies, no patient dies from cancer [27, 28]. On the contrary, thyroid carcinomas in elderly patients with distant metastasis show a poorer prognosis, compared with those without it [29]. These findings contradict the idea that thyroid cancer gradually "acquires" malignant characteristics, such as the ability of invasion or metastasis, later on during repeated proliferation over decades.

The most important point to be discussed when we consider multi-step carcinogenesis is whether "progression" actually occurs in thyroid cancer. Assuming that small and "benign" thyroid cancers turn into more aggressive ones, why didn't a single patient die from thyroid cancer during the observation trial of PMCs? [14] Why did the preventive surgery for PMCs in Korea not contribute to a reduction in thyroid cancer mortality? [5]

The recently emerging model of thyroid carcinogenesis is fetal cell carcinogenesis (Fig. 1C) [30-34]. In fetal cell carcinogenesis, the origin of thyroid cancer is not thyrocytes, but the remnants of fetal thyroid cells existing during the fetal period. The initiation of thyroid cancer occurs during the infantile period. It is not possible to induce thyroid cancer in adults, since the targets for carcinogenesis have already been eliminated.

There are two types of thyroid cancer. Mature cancers arise from differentiated fetal cells, thyroblasts. Mature cancers start growing immediately after their initiation and show rapid growth in young patients.
However, they stop growing in middle age due to their limited ability to proliferate. Mature cancers in the fetal cell carcinogenesis theory can be regarded as self-limiting cancers in the thyroid cancer trilogy. Another type of cancer, originating from undifferentiated thyroid stem cells is called immature cancer. Immature cancers remain silent for decades after the initiation and suddenly start proliferating after middle age. Due to their perennial capacity to proliferate, they are associated with a poor prognosis and can be lethal.

Mature cancers are not always innocent, because some mature cancers creating environmental conditions preferable for the growth of thyroblasts, can kill patients before they stop proliferating. However, most of them present as PMCs after middle age and they do not turn into immature cancers. The initial growth rate of immature cancers is very high and they frequently cause metastasis. As a consequence, they have little chance to be found as PMCs in the absence of metastasis, which can be regarded as subjects for the observation trials. Such a consideration may explain the reason why no patient died from thyroid cancer during the observation trials of PMCs. Thus, mature and immature cancers look similar pathologically, but are different in their origins, growth, and clinical course.

A differentiated cell turns into an undifferentiated one in multi-step carcinogenesis, whereas it is opposite in fetal cell carcinogenesis. As described in the previous section, there is no clear evidence that a differentiated thyroid cancer cell turns into an undifferentiated one. Todaro et al. found that some differentiated thyroid cancer tissues contain a small number of undifferentiated cells expressing a stem cell marker, aldehyde dehydrogenase 1 (ALDH1) (Fig. 3) [35]. The expression of ALDH1 was negatively correlated with a differentiation marker, thyroglobulin (Tg). They separated ALDH1positive and -negative cells, and then the cells were injected into nude mice. Only ALDH-positive cells formed tumors. Immunohistochemical examinations showed that the formed tumors expressed $\mathrm{Tg}$ but not ALDH1. These findings proved, at least in this model, that differentiated cells do not turn into undifferentiated cells to form tumors, whereas undifferentiated cells can form tumors by changing into differentiated cells.

Although the fetal cell carcinogenesis model may help to explain much clinical and experimental evidence, there is an important question that needs to be answered: Why do thyroid stem cells, the origin 


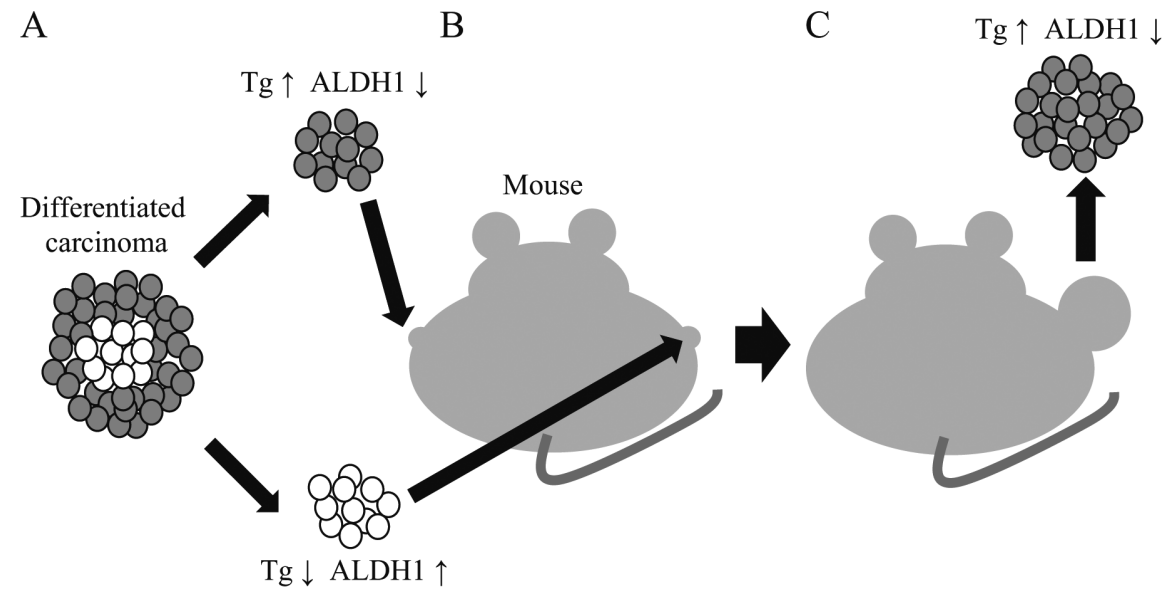

Fig. 3 Implantation of thyroid cancer cells into a nude mouse

Dispersed cancer cells from a differentiated thyroid carcinoma were separated by the expression of ALDH1 (A), then they were implanted at different sites in a nude mice (B). A tumor grew only from ALDH1-positive cells (C). This tumor showed a low expression of ALDH1 and an elevated expression of Tg.

of immature cancers, remain silent for decades? Thyroblasts, which are the origin of mature cancers, are likely to be eliminated before adolescence, since the radiation-induced cancer risk is limited in young children. If this is true, the initiation of immature cancers should occur in the infantile period since thyroid stem cells are eliminated before thyroblasts. However, lethal thyroid cancers do not appear clinically until middle age. We do not have any information to help answering this question at present. If immature cancers are closely related to thyroid stem cells, this phenomenon may be caused by the fundamental nature of thyroid stem cells, whose properties are mostly unknown.

\section{Predicted outcome of the Fukushima Health Management Survey}

After the Fukushima-Daiichi nuclear plant accident, repeated ultrasonographic screening involving about 300,000 children was started in Fukushima Prefecture, Japan. So far, 113 and 25 children have suspected thyroid cancer based on the first and second screenings, respectively [8]. Using each model of the natural history of thyroid cancer, we can predict the outcome of this survey (Fig. 4 and Table 1). Furthermore, we may be able to choose the model that is likely to be correct in the very near future by comparing the data from the first to third screenings.

The following discussion assumes that almost all the children will continue to undergo the ultrasonographic screening. In the late-onset multi-step carcinogenesis model, few thyroid cancers are detected in the first screening, and if all existing thyroid cancers in children are detected in the first screening, no cancer will be detected in the second or third screening since new thyroid cancers will not arise until the children grow up and enter middle age. When the detected cancers are removed by surgery, such surgeries will reduce the mortality associated with thyroid cancer in the future, since these cancers turn into more aggressive ones after middle age.

In the early-onset multi-step carcinogenesis, a moderate number of thyroid cancers are detected in the first screening. A small and the same number of thyroid cancer will be detected in the second and third screenings, since the prevalence of thyroid cancer increases gradually according to the age. Surgery to remove the detected cancers will help to reduce the associated mortality in the future, since these cancers turn into more aggressive ones after several decades.

Showing a marked contrast to the above two models, the outcome in the fetal cell carcinogenesis model is very poor. A large number of thyroid cancers are detected in the first screening. All of them are mature cancers and most will become PMCs in the future. A large number of thyroid cancers will be detected in the second screening, since more thyroid cancers will grow to a size detectable by ultrasonography when the average age increases. More thyroid cancers will be detected in the third screening, since the prevalence 


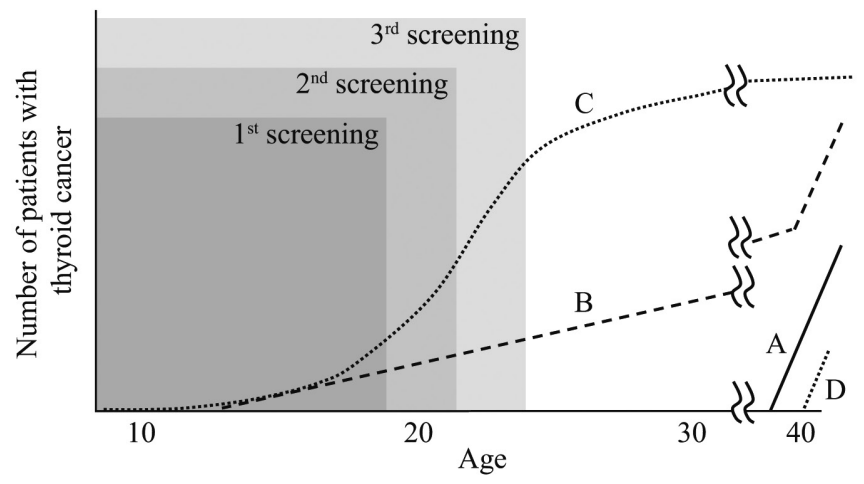

Fig. 4 The Fukushima Health Management Survey and the three models of natural history of thyroid cancer

A, the late-onset multi-step carcinogenesis model; $\mathbf{B}$, the early-onset multi-step carcinogenesis model; $\mathbf{C}$, mature cancers in the fetal cell carcinogenesis model; and $\mathbf{D}$, immature cancers in the fetal cell carcinogenesis model.

Table 1 Predicted outcome of the Fukushima Health Management Survey

\begin{tabular}{|c|c|c|c|c|}
\hline \multirow[b]{2}{*}{ Model } & \multicolumn{3}{|c|}{ Number of detected thyroid cancer patients } & \multirow{2}{*}{$\begin{array}{l}\text { Future } \\
\text { mortality }\end{array}$} \\
\hline & $1^{\text {st }}$ screening & $\begin{array}{l}\text { Comparison of } \\
1^{\text {st }} \text { and } 2^{\text {nd }}\end{array}$ & $\begin{array}{c}\text { Comparison of } \\
2^{\text {nd }} \text { and } 3^{\text {rd }}\end{array}$ & \\
\hline Late-onset multi-step carcinogenesis & Very small (almost zero) & $1^{\text {st }} \gg>2^{\text {nd }}$ & $2^{\text {nd }}=3^{\text {rd }}$ & $\downarrow$ \\
\hline Early-onset multi-step carcinogenesis & Moderate & $1^{\text {st }}>2^{\text {nd }}$ & $2^{\text {nd }}=3^{\text {rd }}$ & $\downarrow \downarrow$ \\
\hline Fetal cell carcinogenesis (mature cancer) & Large & $1^{\text {st }}>2^{\text {nd }}$ & $2^{\text {nd }}<3^{\text {rd }}$ & $\rightarrow$ \\
\hline
\end{tabular}

of thyroid cancer increases steeply from the age of 15 to 30 (Fig. 2). Surgery to remove these cancers will not contribute to reduce thyroid cancer mortality since these are mature cancers which are not lethal. Lethal immature cancers appear after middle age. Thus, in the fetal cell carcinogenesis model, the Fukushima Health Management Survey is predicted to lead to marked overdiagnosis and overtreatment.

\section{Clinical management of thyroid cancers based on the new concept of their natural history}

Apart from the discussion about multi-step and fetal cell carcinogenesis, the thyroid cancer trilogy revealed the existence of two distinct types of thyroid cancer, self-limiting and lethal cancers (Fig. 5). Such a consideration is consistent with the finding that thyroid cancers in those under the age of 20 and over 60 are aggressive compared with those in middle age, since self-limiting and lethal cancers proliferate rapidly in young and elderly patients, respectively [36].

One of the most surprising findings in the Fukushima Health Management Survey was that the mean size of papillary carcinomas detected in the first screening was $1.42 \mathrm{~cm} \mathrm{[8].} \mathrm{Thus,} \mathrm{the} \mathrm{majority} \mathrm{of} \mathrm{the} \mathrm{tumors}$ were macro carcinomas but most of them could not detected without ultrasound. Most of these tumors are regarded as self-limiting cancers, which are considered to stop growing at middle age and then become PMCs. However, when taking into account the fact that juvenile thyroid cancers are very rare, it is likely that a large number of these tumors might reduce in size toward middle age. Thus, early detection or surgery results in marked overdiagnosis and overtreatment. Strict restrictions may be required in the indications for surgery for a juvenile thyroid cancer found incidentally by ultrasonography. Furthermore, thyroid screening of the young should be restricted, since it is likely to result in overdiagnosis.

Thyroid cancers in the elderly are a mixture of self-limiting and lethal cancers, and it is not easy to distinguish between them. The data from the observation trial of PMCs suggested that self-limiting cancers do not grow in patients over the age of 60. Thus, when a papillary carcinoma in patients over the age of 60 shows definite growth, it is likely to be a lethal cancer. In the elderly, ultrasonographic screening can be beneficial when it is used for the early detection of lethal cancer; however, immediate surgery after detection is not recommended, especially for PMCs. Instead, repeated examinations by ultrasonography, 


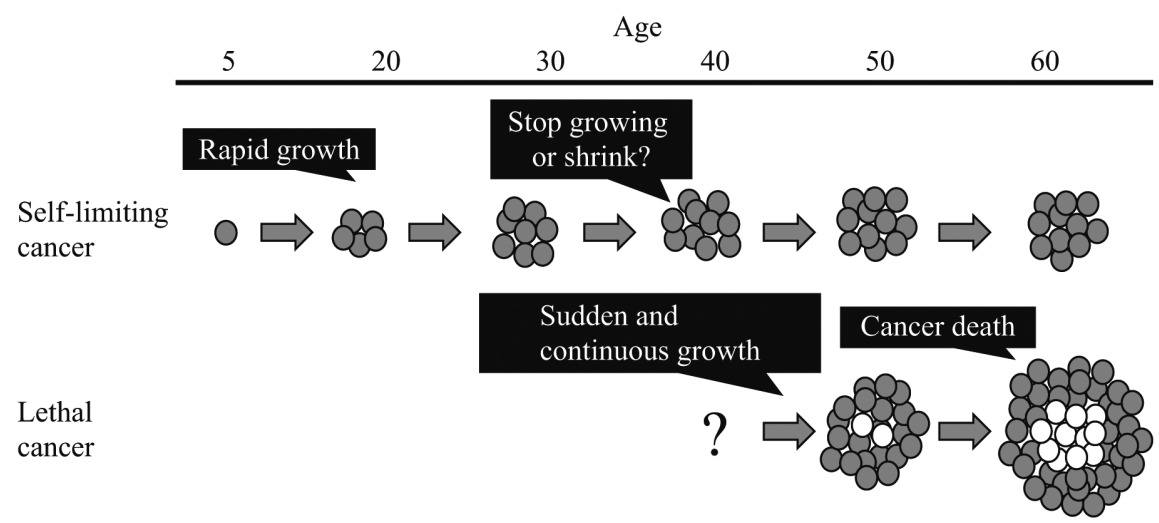

Fig. 5 Natural history of thyroid cancer suggested by the thyroid cancer trilogy

Self-limiting cancers arise in the infantile period and then undergo rapid but limited growth. Most of them stop growing, then turn into PMCs. Lethal cancers appear suddenly after middle age and undergo rapid and unlimited proliferation to cause cancer death.

calculating the growth rate, should be considered. However, tumors with an evident metastasis or extra-thyroidal invasion should be excluded from such an observation trial.

\section{Future prospects}

The thyroid cancer trilogy revealed the existence of self-limiting cancer for the first time in human history. For the past several decades, researchers and clinicians have considered that cancers undergo progression; thus, eventually, all cancers are recommended for early detection and surgery. However, as for self-limiting cancers, such early actions do not lead to a favorable result.
When the excellent prognosis associated with thyroid cancer is taken into account, self-limiting cancers may occupy the majority of all thyroid cancer. Thus, it is easy to imagine that a considerable number of thyroid cancer patients have undergone unnecessary surgery, especially young patients. In the future, surgical resection will be limited to that for lethal cancers and, if necessary, for fast-growing self-limiting cancers. However, at present, there is no known indicator to distinguish self-limiting cancers from lethal cancers except for the patient's age. The detection of thyroid cancer stem cells in tumor tissues can be one of the ways to select thyroid cancers associated with a poor prognosis, although such a technique has yet to be established $[37,38]$.

\section{References}

1. Kondo T, Ezzat S, Asa SL (2006) Pathogenetic mechanisms in thyroid follicular-cell neoplasia. Nat Rev Cancer 6: 292-306.

2. Nikiforov YE, Nikiforova MN (2011) Molecular genetics and diagnosis of thyroid cancer. Nat Rev Endocrinol 7: 569-580.

3. Frazell EL, Foote FW Jr (1949) The natural history of thyroid cancer; a review of 301 cases. J Clin Endocrinol Metab 9: 1023-1030.

4. Burn JI, Taylor SF (1962) Natural history of thyroid carcinoma. A study of 152 treated patients. $\mathrm{Br} M e d J 2$ : 1218-1223.

5. Ahn HS, Kim HJ, Welch HG (2014) Korea's thyroidcancer "epidemic"--screening and overdiagnosis. $N$ Engl J Med 371: 1765-1767.

6. Ahn HS, Welch HG (2015) South Korea's Thyroid-
Cancer "Epidemic"--Turning the Tide. $N$ Engl J Med 373: 2389-2390.

7. Wada N, Duh QY, Sugino K, Iwasaki H, Kameyama K, et al. (2003) Lymph node metastasis from 259 papillary thyroid microcarcinomas: frequency, pattern of occurrence and recurrence, and optimal strategy for neck dissection. Ann Surg 237: 399-407.

8. Suzuki S (2016) Childhood and adolescent thyroid cancer in Fukushima after the Fukushima Daiichi nuclear power plant accident: 5 years on. Clin Oncol ( $R$ Coll Radiol) 28: 263-271.

9. Nikiforov Y, Gnepp DR, Fagin JA (1996) Thyroid lesions in children and adolescents after the Chernobyl disaster: implications for the study of radiation tumorigenesis. J Clin Endocrinol Metab 81: 9-14.

10. Holm LE, Dahlqvist I, Israelsson A, Lundell G (1980) 
Malignant thyroid tumors after iodine-131 therapy: a retrospective cohort study. $N$ Engl J Med 303: 188-191.

11. Jhiang SM, Sagartz JE, Tong Q, Parker-Thornburg J, Capen CC, et al. (1996) Targeted expression of the ret/ PTC1 oncogene induces papillary thyroid carcinomas. Endocrinology 137: 375-378.

12. Charles RP, Iezza G, Amendola E, Dankort D, McMahon M (2011) Mutationally activated BRAF(V600E) elicits papillary thyroid cancer in the adult mouse. Cancer Res 71: 3863-3871.

13. Shimamura M, Nakahara M, Orim F, Kurashige T, Mitsutake N, et al. (2013) Postnatal expression of BRAFV600E does not induce thyroid cancer in mouse models of thyroid papillary carcinoma. Endocrinology 154: 4423-4430.

14. Ito Y, Miyauchi A, Kihara M, Higashiyama T, Kobayashi $\mathrm{K}$, et al. (2014) Patient age is significantly related to the progression of papillary microcarcinoma of the thyroid under observation. Thyroid 24: 27-34.

15. Sciuto R, Romano L, Rea S, Marandino F, Sperduti I, et al. (2009) Natural history and clinical outcome of differentiated thyroid carcinoma: a retrospective analysis of 1503 patients treated at a single institution. Ann Oncol 20: 1728-1735.

16. Neff RL, Farrar WB, Kloos RT, Burman KD (2008) Anaplastic thyroid cancer. Endocrinol Metab Clin North Am 37: 525-538, xi.

17. Autopsy database of the Japanese Society of Pathology (http://pathology.or.jp/kankoubutu/JSP-hyou.html).

18. Williams D (2015) Thyroid growth and cancer. Eur Thyroid J 4: 164-173.

19. Saad AG, Kumar S, Ron E, Lubin JH, Stanek J, et al. (2006) Proliferative activity of human thyroid cells in various age groups and its correlation with the risk of thyroid cancer after radiation exposure. $J$ Clin Endocrinol Metab 91: 2672-2677.

20. Viglietto G, Chiappetta G, Martinez-Tello FJ, Fukunaga FH, Tallini G, et al. (1995) RET/PTC oncogene activation is an early event in thyroid carcinogenesis. Oncogene 11: 1207-1210.

21. Fagin JA, Matsuo K, Karmakar A, Chen DL, Tang SH, et al. (1993) High prevalence of mutations of the p53 gene in poorly differentiated human thyroid carcinomas. J Clin Invest 91: 179-184.

22. Kroll TG, Sarraf P, Pecciarini L, Chen CJ, Mueller E, et al. (2000) PAX8-PPARgammal fusion oncogene in human thyroid carcinoma [corrected]. Science 289: 1357-1360.

23. Kimura ET, Nikiforova MN, Zhu Z, Knauf JA, Nikiforov YE, et al. (2003) High prevalence of BRAF mutations in thyroid cancer: genetic evidence for constitutive activation of the RET/PTC-RAS-BRAF signaling pathway in papillary thyroid carcinoma. Cancer Res 63: $1454-1457$
24. Namba H, Nakashima M, Hayashi T, Hayashida N, Maeda S, et al. (2003) Clinical implication of hot spot BRAF mutation, V599E, in papillary thyroid cancers. $J$ Clin Endocrinol Metab 88: 4393-4397.

25. Xing M, Westra WH, Tufano RP, Cohen Y, Rosenbaum $\mathrm{E}$, et al. (2005) BRAF mutation predicts a poorer clinical prognosis for papillary thyroid cancer. $J$ Clin Endocrinol Metab 90: 6373-6379.

26. Mitsutake N, Fukushima T, Matsuse M, Rogounovitch T, Saenko V, et al. (2015) BRAF(V600E) mutation is highly prevalent in thyroid carcinomas in the young population in Fukushima: a different oncogenic profile from Chernobyl. Sci Rep 5: 16976.

27. Ito Y, Miyauchi A, Kihara M, Takamura Y, Kobayashi $\mathrm{K}$, et al. (2012) Relationship between prognosis of papillary thyroid carcinoma patient and age: a retrospective single-institution study. Endocr J 59: 399-405.

28. Kowalski LP, Goncalves Filho J, Pinto CA, Carvalho AL, de Camargo B (2003) Long-term survival rates in young patients with thyroid carcinoma. Arch Otolaryngol Head Neck Surg 129: 746-749.

29. Ito Y, Miyauchi A, Ito M, Yabuta T, Masuoka H, et al. (2014) Prognosis and prognostic factors of differentiated thyroid carcinoma after the appearance of metastasis refractory to radioactive iodine therapy. Endocr $J 61$ : 821-824.

30. Takano T, Amino N (2005) Fetal cell carcinogenesis: a new hypothesis for better understanding of thyroid carcinoma. Thyroid 15: 432-438.

31. Takano T (2007) Fetal cell carcinogenesis of the thyroid: theory and practice. Semin Cancer Biol 17: 233-240.

32. Takano T (2014) Fetal cell carcinogenesis of the thyroid: a modified theory based on recent evidence. Endocr J 61: 311-320.

33. Takano T (2014) The basic theory of fetal cell carcinogenesis of the thyroid. J Basic Clin Med 3: 6-11.

34. Takano T (2015) Molecular classification of thyroid tumor: A proposal based on fetal cell carcinogenesis hypothesis. J Basic Clin Med 4: 81-6.

35. Todaro M, Iovino F, Eterno V, Cammareri P, Gambara G, et al. (2010) Tumorigenic and metastatic activity of human thyroid cancer stem cells. Cancer Res 70: 8874-8885.

36. Mazzaferri EL (2000) Long-term outcome of patients with differentiated thyroid carcinoma: effect of therapy. Endocr Pract 6: 469-476.

37. Reya T, Morrison SJ, Clarke MF, Weissman IL (2001) Stem cells, cancer, and cancer stem cells. Nature 414: 105-111.

38. Matsumoto C, Ito M, Yamada H, Yoshida H, Watanabe M, et al. (2013) Preparation of thyroid follicular cells for mRNA quantification after fluorescence-activated cell sorting. Scand J Clin Lab Invest 73: 245-252. 